\title{
Toxicological responses of normal human bronchial epithelium (NHBE) model exposed to settled dust samples from moisture damaged and reference schools
}

Kati Huttunen ${ }^{1,2}$, Anna Wlodarczyk ${ }^{1}$, Zoë Prytherch ${ }^{1}$, Tim Jones ${ }^{3}$, Martin Täubel ${ }^{4}$, Juha Pekkanen $^{4,5}$, Dick Heederik ${ }^{6}$, Jan-Paul Zock ${ }^{7,8,9}$, Anne Hyvärinen ${ }^{4}$, Maija-Riitta Hirvonen ${ }^{2}$, Kelly BéruBé $^{1}$

${ }^{1}$ Cardiff University, School of Bioscience, Cardiff, United Kingdom

${ }^{2}$ University of Eastern Finland, Department of Environmental Science, Kuopio, Finland

${ }^{3}$ Cardiff University, School of Earth \& Ocean Sciences, Cardiff, United Kingdom

${ }^{4}$ Institute for Health and Welfare, Department of Environmental Health, Kuopio, Finland

${ }^{5}$ University of Helsinki, Department of Public Health, Helsinki, Finland

${ }^{6}$ Utrecht University, Institute for Risk Assessment Sciences, Division Environmental Epidemiology, Utrecht, The Netherlands

${ }^{7}$ Centre for Research in Environmental Epidemiology (CREAL), Barcelona, Spain

${ }^{8}$ Universitat Pompeu Fabra (UPF), Barcelona, Spain

${ }^{9}$ CIBER Epidemiología y Salud Pública (CIBERESP), Madrid, Spain

Exposure to indoor air in moisture damaged buildings is associated with deteriorating respiratory health, assumedly due to emissions from microbial growth and wet building materials. Previous studies of toxicological effects of mouldy house microbes have indicated that inflammation and cell death are important mechanisms.

Aiming to gain further insight into function of respiratory epithelia, we studied the responses of normal human bronchial epithelium (NHBE) model after exposure to settled dust samples $(n=9)$ collected from moisture damaged and reference schools in Spain, The Netherlands and Finland. The results were compared with immunotoxicological potential of the same samples in mouse RAW264.7 macrophage model.

The effects of exposure on the NHBE model was assessed by measuring trans epithelial electric resistance (TEER) of the culture, changes in mucus production (Bradford assay), cell toxicity (ATP assay), cytokine levels in the culture media (ELISA) as well as morphological changes in cultured cells (light and scanning electron microscopy).

The results showed that exposure to dust from moisture damaged schools was capable of inducing both increased TEER and mucus production and in some instances diminished TEER values indicating deterioration of tissue integrity and cell death. Similarly with the results from mouse macrophage model, the samples from The Netherlands and Spain were more toxic compared to samples from Finland. The findings suggest that the defence mechanisms present in respiratory epithelia are activated by dust from moisture damaged buildings, and exposure to high doses may damage the affected lung tissue. 